\title{
Réflexions sur la résurrection de l'escrime médiévale occidentale
}

Méthode, objectifs et limites

Thoughts on the Revival of the European Medieval Swordsmanship. Method, Objectives and Limitations

\section{Sergio Boffa}

\section{OpenEdition}

Journals

Édition électronique

URL : https://journals.openedition.org/tc/8383

DOI : $10.4000 /$ tc. 8383

ISSN : 1952-420X

Éditeur

Éditions de l'EHESS

Édition imprimée

Date de publication : 1 décembre 2014

Pagination : 166-187

ISBN : 978-2-7351-2346-9

ISSN : 0248-6016

Référence électronique

Sergio Boffa, «Réflexions sur la résurrection de l'escrime médiévale occidentale », Techniques \& Culture [En ligne], 62 | 2014, mis en ligne le 01 décembre 2017, consulté le 29 septembre 2022. URL : http:// journals.openedition.org/tc/8383; DOI : https://doi.org/10.4000/tc.8383 


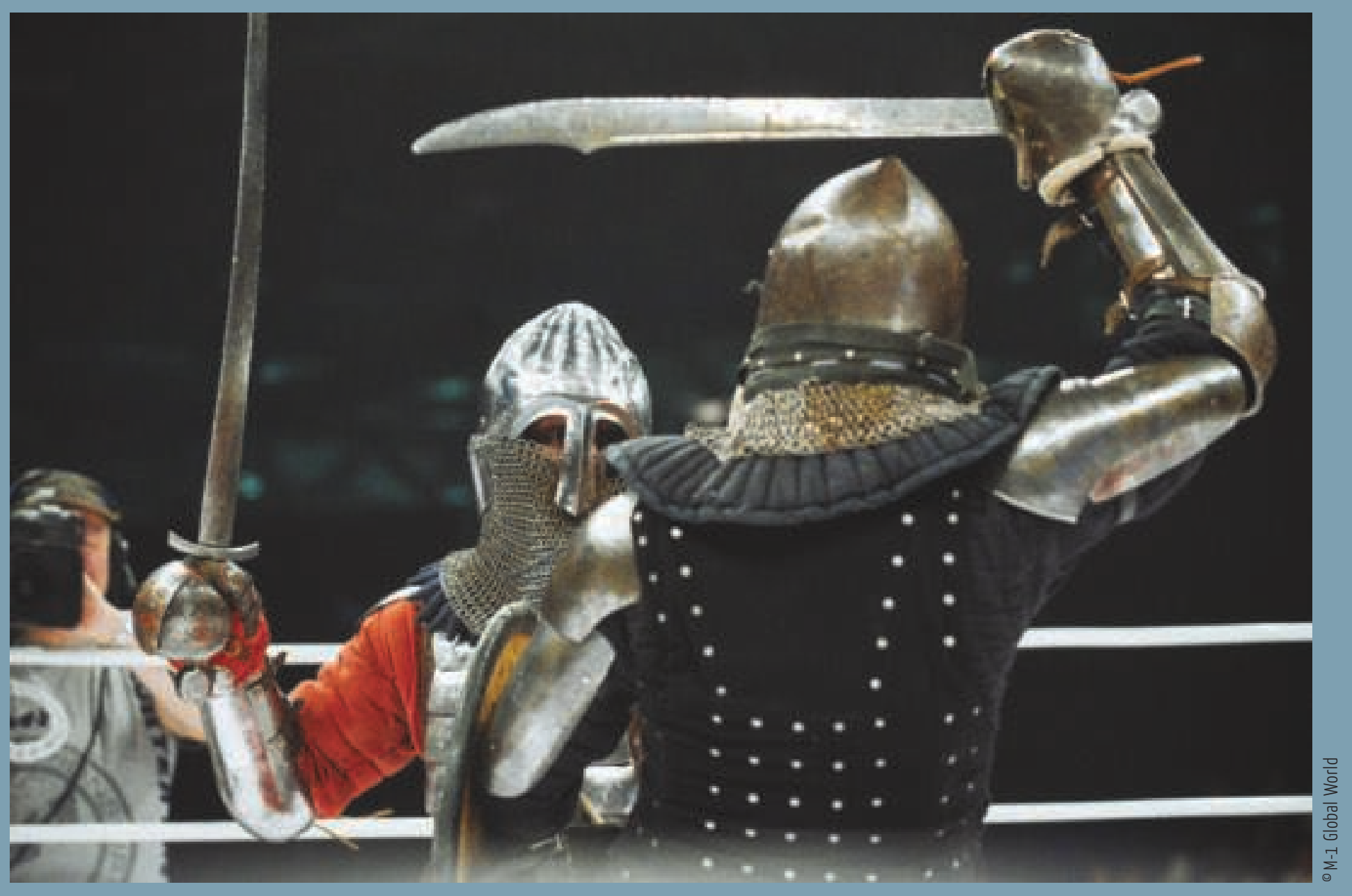




\section{RÉFLEXIONS SUR LA RÉSURRECTION DE L'ESCRIME MÉDIÉVALE}

\section{Méthode, objectifs et limites}

Depuis quelques années, tant en Europe qu'aux États-Unis, un grand nombre de sociétés se sont constituées afin de ressusciter d'anciennes méthodes de combat. Elles étudient ce qu'elles appellent les «Arts martiaux historiques européens » (AMHE) ${ }^{1}$. L'objectif de ces associations n'est pas tant d'acquérir une compréhension générale du combat au corps-à-corps que de redécouvrir et de pratiquer l'enseignement de célèbres maîtres d'armes ayant vécu au Moyen Âge ou plus tard (Jaquet et al. 2015). Cette entreprise est particulièrement difficile à mener. En Occident, la majorité des traditions martiales médiévales s'effacent au XvI ${ }^{e}$ siècle lorsque tant l'art de la guerre que l'armement personnel subissent des changements radicaux (Oakeshott 2000; Capwell 2012). De nos jours, personne ne peut prétendre avoir suivi un enseignement qui se serait transmis de manière ininterrompue au fil des générations. Il s'agit donc bien de ressusciter un savoir oublié ${ }^{2}$

À cette fin, ces chercheurs commencent par se familiariser avec les sources historiques, étudient de manière approfondie l'un ou l'autre texte et se procurent un équipement plus ou moins semblable à celui utilisé par les combattants de jadis. Ensuite, grâce à l'expérimentation, ils espèrent pouvoir donner vie aux informations récoltées lors de leurs analyses. Ce processus, décrit ici dans ses grandes lignes, semble être le moyen idéal pour faire revivre un art martial dont la pratique a cessé depuis plusieurs siècles.

Les membres de ces associations reconnaissent bien quelques limites à leur entreprise, mais cela ne les empêche pas d'utiliser régulièrement des qualificatifs comme «légitime», « historique», «précis» ou « authentique» lorsqu'ils présentent leurs résultats. Ainsi, parmi de nombreux exemples, nous pouvons lire sur le site internet de l'Association for 
Renaissance Martial Arts (ARMA): "The ARMA's efforts are directed toward resurrecting and recreating a legitimate craft of European fighting skills in a manner that is historically valid and martially sound ${ }^{3}$.»

Si le désir de ressusciter une tradition disparue depuis plusieurs siècles est compréhensible et même louable, les écueils qui attendent le chercheur sont nombreux. Suffisamment pour que nous puissions nous interroger sur la faisabilité d'une telle entreprise. Dans cet article nous nous proposons d'essayer de répondre aux problématiques suivantes. Est-il possible de ressusciter un geste martial disparu, à l'aide des seules sources écrites et iconographiques? Quel degré d'historicité ou d'authenticité, pouvons-nous accorder aux résultats obtenus?

\section{Les manuels de combat}

Les manuels de combat ou Fechtbücher sont sans nul doute la source historique la plus importante pour ces chercheurs (Anglo 2000; Boffa 2014; Jaquet 2012). Ces textes, qui appartiennent à la littérature technique (Fachliteratur), nous sont parvenus en assez grand nombre puisque l'ensemble du corpus est estimé à près d'une centaine d'œuvres ${ }^{4}$. Si le plus ancien manuel fut écrit au début du XIV ${ }^{e}$ siècle ${ }^{5}$, la grande majorité de ceux-ci date $d u x^{e}$. L'escrime médiévale se trouve encore décrite dans quelques traités du XvI siècle avant de sombrer dans l'oubli ${ }^{6}$.

Les matières abordées sont très variées: il est question du combat à pied (Fußfechten) ou à cheval (Roßfechten), avec (Harnischfechten) ou sans armure (Bloßfechten). Les armes les plus fréquemment rencontrées sont l'épée, la dague, la hache d'arme, la lance ainsi que certaines armes propres au duel judiciaire. Le combat à mains nues (Ringen) n'est pas oublié et il occupe souvent une part non négligeable de ces traités.

La plupart des manuels sont conservés sous forme manuscrite et les rares imprimés font naturellement leur apparition à la fin du $\mathrm{Xv}^{e}$ siècle $^{7}$. Ces manuels se composent de textes et d'illustrations, dans les proportions suivantes, d'après les estimations de Daniel Jaquet: $47 \%$ de texte et d'illustrations; $36 \%$ de texte seul; $15 \%$ de textes lacunaires et d'illustrations et $2 \%$ d'illustrations seules (Jaquet 2012: 19). De ce fait, retenons que près des deux tiers de ces œuvres sont illustrées: un indice de l'important rôle joué par l'image comme vecteur de transmission du savoir.

Le texte consiste généralement en descriptions plus ou moins détaillées de gardes, de techniques ou de combinaisons de mouvements. Dans la tradition allemande - la tradition dominante-, il se présente souvent sous forme de glose. Dans ce cas, un titre introduit la technique; puis, quelques vers (Merkverse), extraits de la théorie du maître d'armes à l'origine de cette tradition, décrivent l'action; enfin, l'auteur explicite ces quelques vers afin de communiquer sa propre compréhension du mouvement. Selon les auteurs, la description technique peut varier de quelques mots (Hans Talhoffer) à un paragraphe complet (Johannes Lecküchner). La présence ou non d'illustration et les variations, parfois très importantes, du volume du texte impliquent naturellement que tous les manuels de combat ne sont pas égaux face à l'interprétation. Une première constatation s'impose: certains textes resteront à jamais sibyllins. 


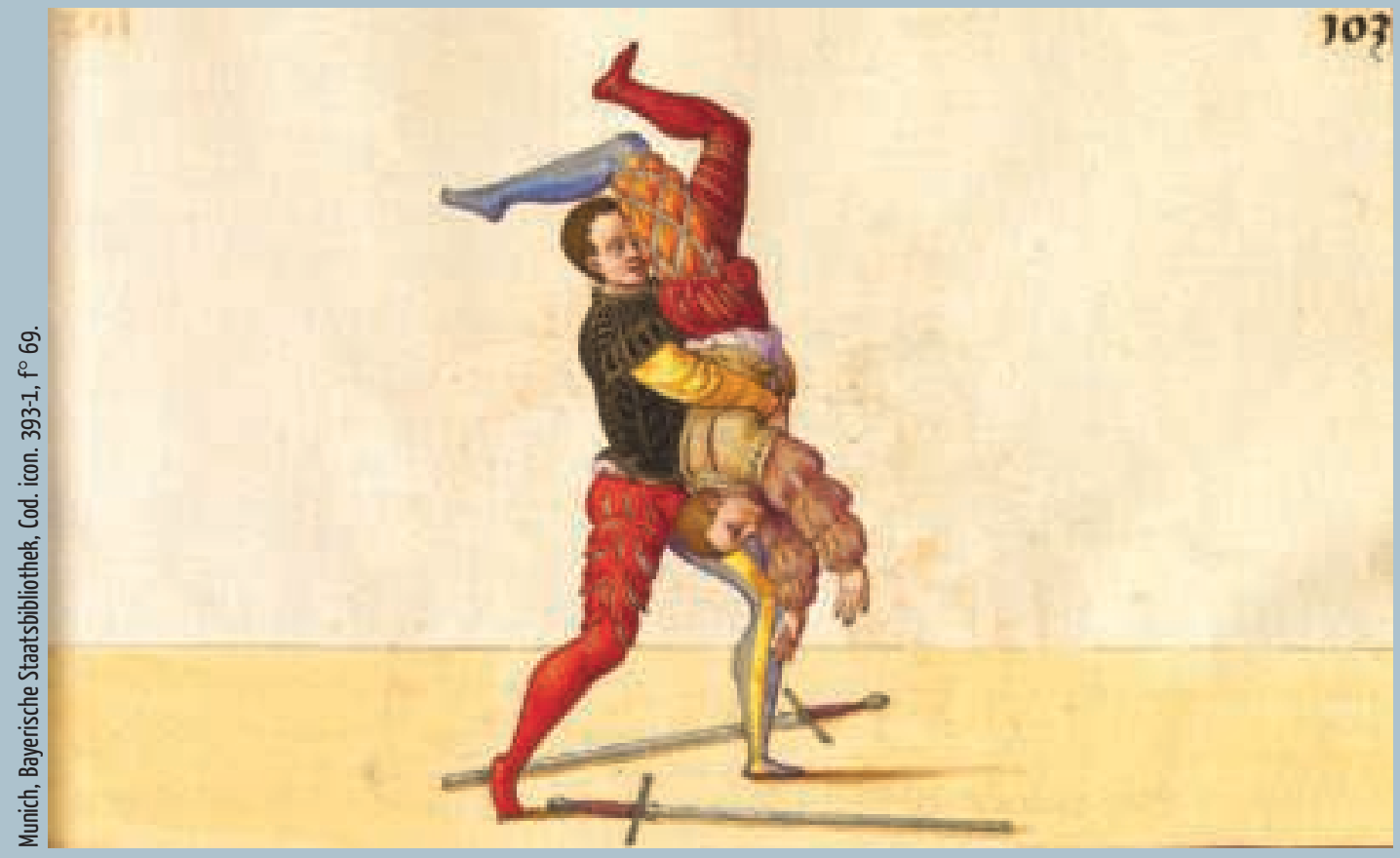

Paul Hector Mair, De Arte Athletica

Nous laissons au lecteur le soin d'imaginer quelle technique de combat peut conduire à une telle position.

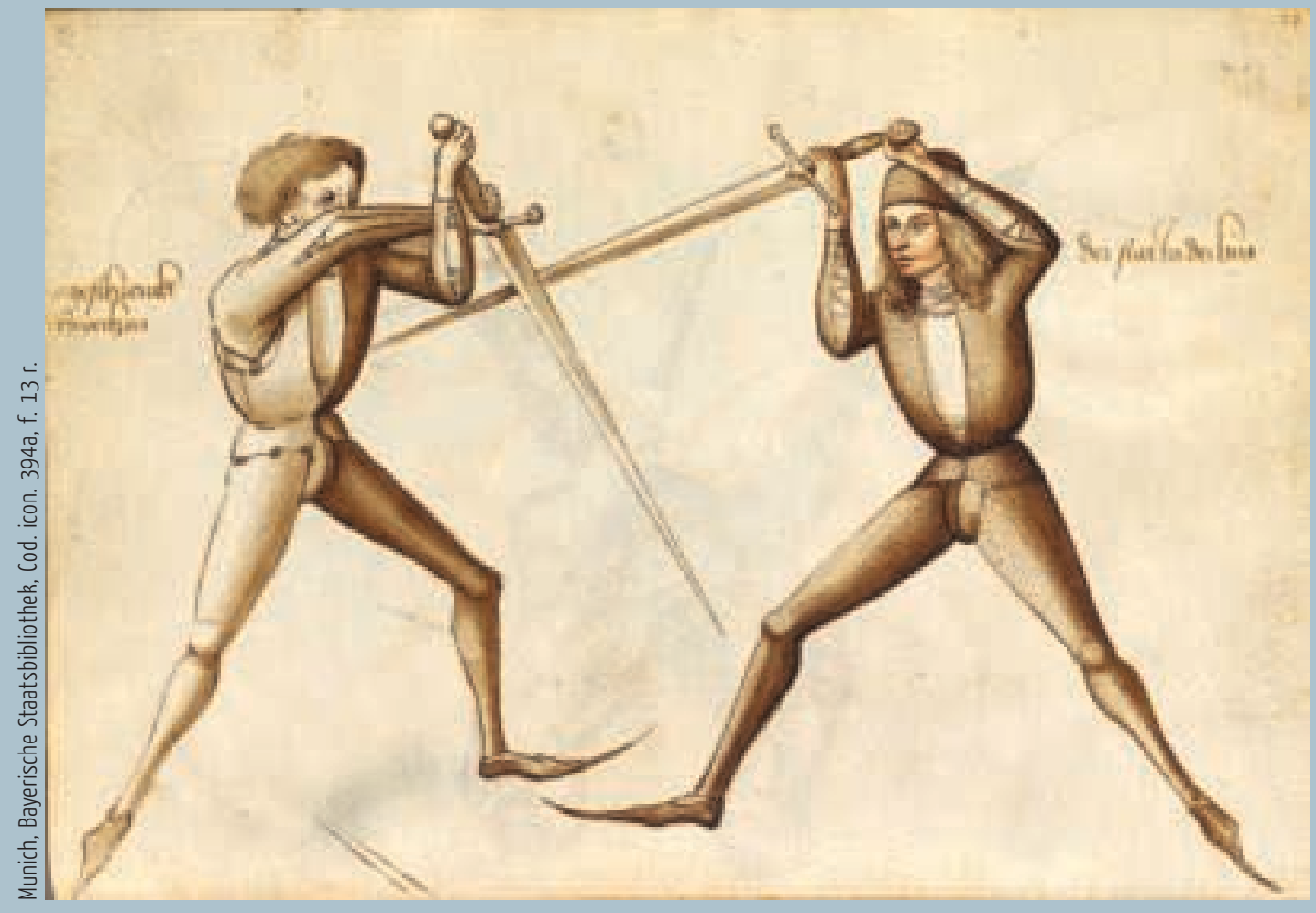

Duel à l'épée longue

L'enseignement de Hans Talhoffer ( $\mathrm{xv}^{\mathrm{e}}$ siècle) se trouve conservé dans plusieurs manuscrits datés du milieu du xve siècle. Leur particularité est d'avoir un texte extrêmement concis et sibyllin. La technique représentée sur ce folio est décrite ainsi : « Die geschrenckt Der stat in der Hut » soit: « La faiblesse raccourcie $\|$ est en garde ». 


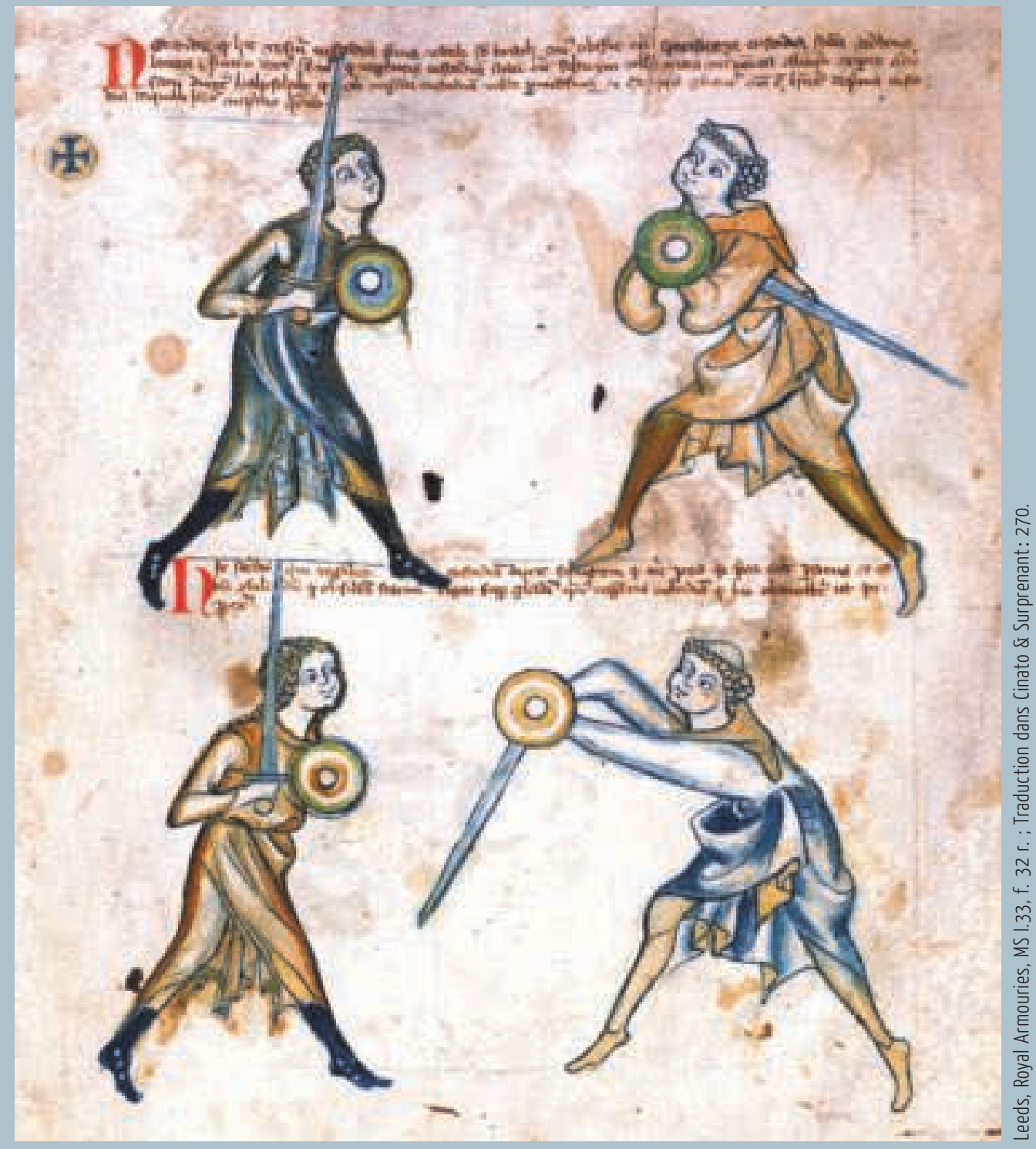

Combat entre un novice et une jeune fille

Le Leeds, Royal Armouries, MS I.33 est le plus ancien manuel de combat à nous être parvenu (fin xille-début xve siècles). La matière traitée dans cette œuvre se limite au combat à l'épée et au petit bouclier. Le texte est en latin, un fait assez rare, mais certains termes techniques sont en langue vernaculaire. C'est le cas dans la seconde légende: Hic sacerdos qui regebat custodiam ducit schutzin, quod erit pro eo quia prior erat paratus. Et est bene consulendum quod obsidens statim ligat super gladium ipsius regentis custodiam, quod hic obmittitur ut patet per exemplum ; « Le prêtre qui dirigeait la garde exécute ici la protection (schutzin), pour ce qu'il était prêt le premier. Et il est bien à conseiller que celui qui assiège fasse aussitôt un liage par-dessus l'épée de celui qui dirige la garde, laquelle action, comme le montre l'exemple est omise dans le cas présent. » 


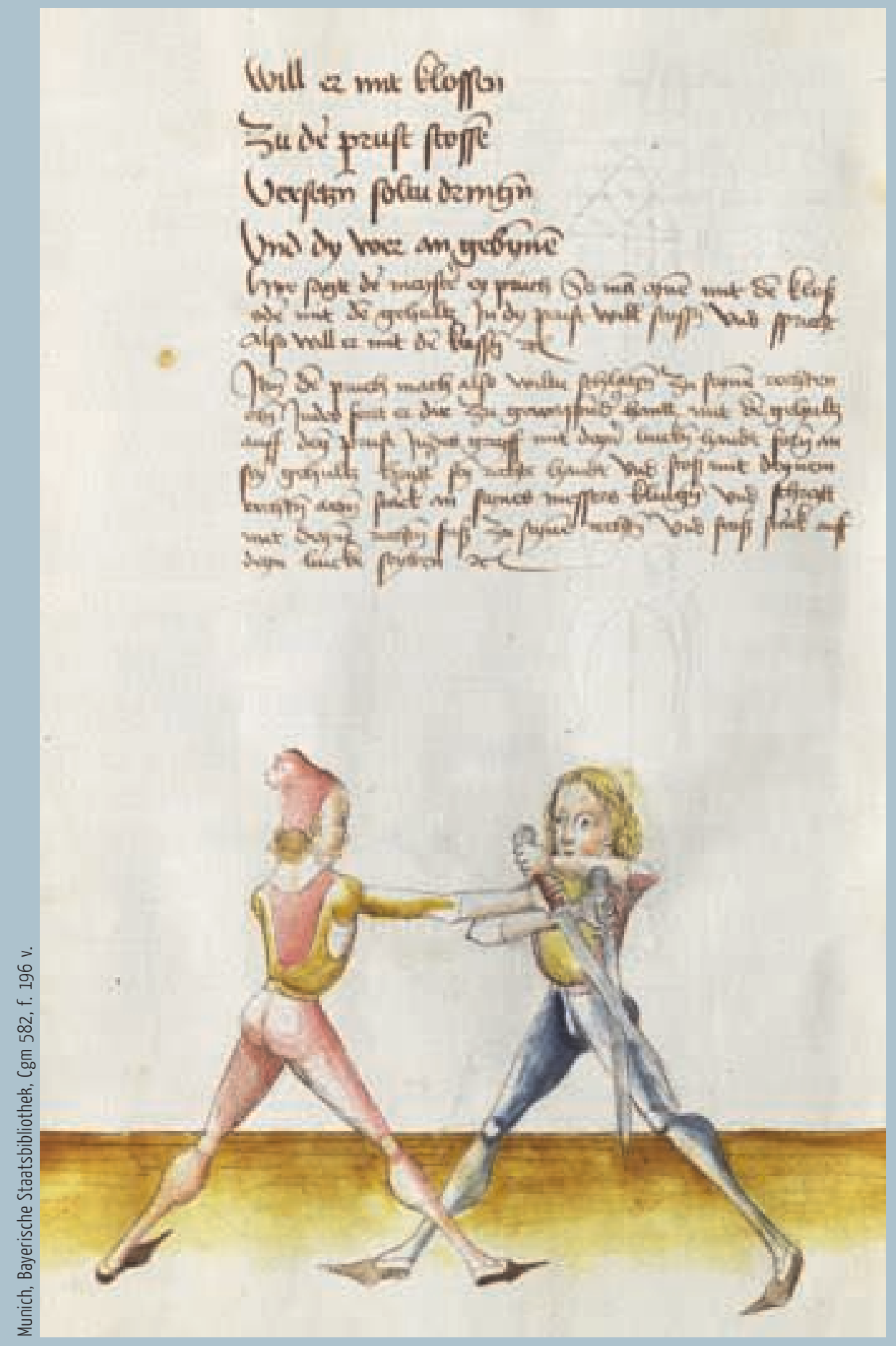

\section{Combat au fauchon ou Lange Messer}

Johannes Lecküchner (seconde moitié du xve siècle) s'est intéressé au maniement du Langes Messer, une épée à large lame et à un seul tranchant. Ses deux traités (1478 et 1482) sont très détaillés. Les descriptions, replacées dans l'ensemble technique du traité, sont assez précises pour que le lecteur puisse se faire une idée réelle du mouvement à exécuter. 


\section{Quelques définitions: mouvement, technique, style et curriculum}

Avant de continuer, il est nécessaire de clarifier plusieurs concepts utilisés dans notre article. Il est important de bien différencier le mouvement de la technique, de comprendre ce qu'est le style personnel d'un maître d'armes et de déterminer l'étendue de son art (son curriculum).

Nous appelons mouvement une action simple ou complexe qui ne constitue pas une réponse directe à une geste de l'adversaire: prendre une garde ou effectuer un déplacement par exemple. Nous considérons aussi comme mouvement toute technique exécutée seul; ce qui arrive lorsqu’on désire la répéter un grand nombre de fois afin d'en maîtriser la mécanique corporelle ${ }^{8}$ ou lorsqu'on s'entraîne à la manière de la shadow boxing. Dans ces conditions, de nombreux aspects liés à l'exécution d'une technique sont absents.

La technique est le mouvement mis en situation, c'est-à-dire lorsqu'il est exécuté en fonction des agissements d'un partenaire. Alors, de nouveaux éléments entrent en jeu. Des facteurs spatio-temporels d'une part, ils comprennent la gestion de la distance de combat, le choix du moment où l'action sera initiée ainsi que la détermination de la vitesse et de la puissance nécessaires à son exécution. Des facteurs psychologiques d'autre part, comme la force mentale qu'il faut posséder pour accepter un combat mortel ou le courage qu'il faut détenir pour lancer une attaque contre un adversaire fort et déterminé. L'importance de ces derniers éléments n'est pas à négliger, toute faiblesse mentale peut ruiner des années d'entraînement ${ }^{9}$. La technique (de combat) est ainsi une action bien plus complexe que le mouvement ${ }^{10}$ (Ohya 2000: 39-65).

La manière d'exécuter une ou plusieurs techniques engendre le style du protagoniste. Il est aisé de confirmer que deux personnes, quel que soit leur niveau d'habilité, n'exécuteront pas similairement un même mouvement ou une même technique ${ }^{11}$. Les différences peuvent être dérisoires - de l'ordre de quelques centimètres ou de quelques degrés - ou significatives. Ce n'est pas le plus important, mais l'existence même de différents styles, quels qu'ils soient, doit être soulignée.

L'étendue de l'art d'un maître d'armes ou d'une tradition est simplement constituée par l'ensemble des techniques proposées par ledit maître ou ladite tradition ${ }^{12}$. Selon les époques et les lieux, le curriculum peut être accru (environs deux cents techniques pour Johannes Lecküchner) ou restreint (une dizaine dans le Man yt $\mathrm{Wol})^{13}$, plutôt offensif (Johannes Liechtenauer) ou défensif (Fiore dei Liberi), en faveur de la maîtrise d'une seule arme (Johannes Lecküchner) ou de plusieurs (la plupart des maîtres allemands), etc. À première vue, ces précisions peuvent paraître futiles, mais elles acquièrent toute leur importance lorsqu'il s'agit de déterminer l'ampleur des objectifs atteints par les tentatives de reconstructions. 


\section{La difficulté de concevoir une méthode de travail}

La lecture d'un manuel, dont l'auteur est un tant soit peu prolixe, permet de découvrir les idées et les concepts qui structurent son enseignement. Cette première approche est fondamentale, mais reste toute théorique. À ce stade, nous sommes encore loin d'avoir reconstitué son arsenal de techniques de combat. Pour cela, il est nécessaire d'élaborer une méthode de travail rigoureuse, scientifique diront certains. Il ne suffit évidemment pas d'affronter un partenaire les armes à la main pour y prétendre:

«Playing with a sword simulator, in a modern-day outfit, with a friendly partner (or against an adversary in a competition), applying training based on interpretations, presented by a self-proclaimed (or recognized by his peer as such) instructor, or out of a web-published translation of source material, is a common feature of HEMA practice. It is not scientific research; it may be element phases of a research project. It is not experimenting HEMA; it is experiencing HEMA. It is not restoring a documented historical martial praxis; it may be considered a reviving attempt as argued by Unesco standards, or more commonly named "re-creating" ${ }^{14}$ " (Jaquet et al. 2015: 16-20).

En travaillant ainsi, ces pratiquants développent davantage une interprétation personnelle du texte qui leur sert de guide qu'ils ne participent à la résurrection du savoir qui se trouve conservé. Les tentatives élaborées pour ressusciter les arts martiaux occidentaux ne manquent pourtant pas, et certaines sont surprenantes. Jamie Acutt a par exemple développé ce qu'il appelle la Paleoeskirmological Method, curieusement et bien qu'il prétende s'intéresser à l'escrime historique; il n’hésite pas à considérer les sources anciennes comme négligeables:

«The limitation with the ARMA Method is that it interprets that which has historical evidence, and therefore can only re-create that which has been documented. The Paleoeskirmological Method, on the other hand, is based upon the logical purpose of the original fighting arts meaning that our understanding of the use of anachronistic weaponry is direct and not via the fossilised words of an historical source. Experience and logical understanding of combat using an anachronistic weapon - directed by function and a determination to solve the problems of risk and control in interpersonal combat using that weapon - first and foremost, then approaching historical records to source correlates. Of course, this source does have its importance, but in Paleoeskirmology, the historical source is always secondary as a route to true knowledge than our own experience itself. Therefore, with a solid foundation in Eskirmological Principles, Paleoeskirmology represents the closest possible, and logical, source for the re-creation of long-extant arms and armour ${ }^{15}$. »

Cet exemple, extrême il est vrai, montre clairement les dérapages possibles. Cependant tous les chercheurs ne sont pas aussi farfelus. Toutefois, la majorité d'entre eux se contente malheureusement de présenter le résultat de ses recherches et omet de décrire la méthode développée pour y parvenir. Et lorsqu'il est question de méthode, la plupart énonce quelques principes généraux et souligne la difficulté de la tâche (Windsor 2005: 29-37; Hand 2005: 39-47; Rubboli 2011 : 77-90; Malipiero 2011 : 109-116). Enfin, de temps à autre, quelques-uns de ces spécialistes nous dévoilent utilement leur secret (Alderson 2010: 251-286; Lindholm 2003: 209-211; Colciago 2014: 119-161). C'est le cas de Bartłomiej Walczak (2006: 121-142) qui a développé la méthode «AGISE» acronyme de Analysis, Grouping, Interpolation, Synthesis, Extrapolation. 
Le chercheur commence par essayer de découvrir comment une technique était exécutée. Pour cela, il la décompose en mouvements élémentaires (phase d'analyse). Ensuite, il essaie de trouver des techniques similaires à celle qu'il étudie (phase de regroupement). Une fois la technique placée dans un ensemble homogène, il recherche le principe fondamental à l'origine de ces techniques (phase d'interpolation). La découverte de ce principe permet au chercheur d'étudier ou d'élaborer certaines variantes techniques. Il revoit alors l'ensemble du savoir qu'il a accumulé afin de vérifier sa cohérence. C'est également le moment où il vérifie par la pratique si la technique telle qu'elle a été reconstituée est utilisable en situation de combat (phase de synthèse). Pour terminer, il confronte les résultats obtenus grâce à ce processus avec d'autres sources historiques (phase d'extrapolation).

La méthode AGISE ne manque pas d'intérêt. Néanmoins, malgré tous ses mérites, elle comporte certaines limitations. En effet, la phase d'analyse est particulièrement délicate à mener. Le bon déroulement de la recherche et la valeur des résultats dépendent fortement de la qualité des informations extraites du manuel. Il n'existe pourtant aucune marche à suivre reconnue pour nous guider dans cette étape; ce n'est guère surprenant étant donné le manque d'homogénéité des textes qui composent le corpus de manuels. Les chercheurs sont donc livrés à eux-mêmes. Ainsi, nous ne sommes pas étonnés de voir Grzegorz Żabiński, Russel A. Mitchell et Falko Fritz travailler de la manière suivante:

«The interpreters within this work assume that the fencing actions described and depicted in Lecküchner's work derive from the following base:

1. Competent illustrator [...]

2. Floating footwork [...]

3. No centerline $[\ldots]$

4. Power generation $[\ldots]$

Any difference in base assumptions from this by interpreters of the text will result in a difference interpretation of the action described below. This does not mean that one set of assumptions is as valid as any other, but in the opinion of the authors, deviations from these assumptions detailed above will make it difficult to explain how certain techniques could be translated in detailed movements» (Żabiński et al. 2012: 30-31).

Le procédé déconcerte: conduire une recherche sur la base de quatre postulats difficiles à vérifier ou dont la véracité reste à démontrer semble douteux. Peut-on qualifier cette méthode de scientifique et quel crédit peut-on accorder aux résultats obtenus par celle-ci? Des faiblesses méthodologiques similaires s'observent bien trop souvent.

\section{Les principaux problèmes}

Lélaboration d'une méthode de travail fiable n'est pas la seule difficulté à laquelle le chercheur doit faire face. Il n'est évidemment pas question de développer ici tous les problèmes qui surgissent lorsque l'on désire exploiter un manuscrit médiéval (étude du codex, identification du texte et de son auteur, datation de l'œuvre, établissement du stemma codicum, etc.); tout cela a déjà fait l'objet de nombreux ouvrages techniques de qualité. Nous nous concentrerons uniquement sur les obstacles en lien direct avec la source spécifique qu'est le manuel de combat ou avec à l'objet même de la recherche: la technique de combat. 


\section{Les arts martiaux médiévaux européens sont extrêmement complexes}

L'escrime médiévale est souvent présentée comme primitive et brutale (Castle 2003: 13-14; Lacaze 1991 : 24; Cohen 2002: 23). Pourtant il suffit de parcourir un manuel de combat pour comprendre que cette idée est à rejeter définitivement ${ }^{16}$. Plusieurs caractéristiques de l'escrime médièvale ont déjà été mises en évidence; elles soulignent la grande subtilité des arts martiaux européens. Portons brièvement notre attention sur la tradition allemande, initiée par le maitre d'armes Johannes Liechtenauer (xiv siècle), afin d'en avoir un aperçu. Nous découvrons de multiples gardes (Leger), les arcanes des quatre ouvertures ou opportunités (Vier Bloßen), les différentes manières d'utiliser l'épée (Drei Wunder) - c'est-à-dire de piquer (Stich), de trancher (Hau) ou de couper (Schnitt)-, les trois moments de l'attaque (Vor, Nach et Indes), les problèmes de distances (Lenge und $\mathrm{Ma} \beta$ e) ou des notions encore plus abstraites comme le «sentiment» (Fühlen) ${ }^{17}$. Des concepts analogues, ou tout aussi sophistiqués, se distinguent également dans la tradition italienne (Price 2008; Windsor 2012). L'existence d'une théorie aussi complexe atteste que l'escrime médiévale n'était pas seulement un art empirique où l'expérience permettait d'accumuler mouvements et stratagèmes destinés à vaincre l'ennemi. Elle faisait l'objet d'une profonde réflexion qui conduisait à une théorisation des techniques et à l'élaboration de principes généraux, parfois d'une grande finesse. Tout cela rend sa redécouverte des plus excitante, mais aussi des plus difficile.

\section{Quelle est l'escrime transmise par les manuels de combat?}

Il est légitime de s'interroger sur la représentativité de l'escrime décrite dans les manuels. Auparavant, rappelons que la grande majorité de ces œuvres a été produite au Xve siècle. L'équipement offensif et défensif étant en constante évolution, les techniques de combat s'adaptaient invariablement à ces changements afin de préserver toute leur efficacité. Ainsi, nul doute que l'escrime du Bas Moyen Âge soit différente de celle du Moyen Âge central ou du Haut Moyen Âge ${ }^{18}$. Ensuite, la grande majorité des manuels sont issus des terres d'Empire: Allemagne, Italie du nord et Suisse. Aucun texte n'a été retrouvé dans les anciens Pays-Bas, un seul a été découvert pour la France et trois pour l'Angleterre. Pour autant il est impensable que des arts de combat dignes de ce nom n'aient pas été développés dans le reste de l'Europe. Mais, dès lors que nous relevons les différences notables qui existent entre les traditions allemande et italienne, nous comprenons qu'il n'est pas possible d'imaginer qu'une seule et même escrime ait été pratiquée dans tout l'Occident. Un escrimeur anglais, français ou bourguignon ne maniait probablement pas l'épée exactement comme on le faisait dans les terres centrales de l'Empire. Malheureusement, faute de source, ces spécificités régionales nous échappent pour l'instant. Enfin, le contenu des manuels ne semble pas être adapté aux besoins de l'élite guerrière de l'époque: l'homme d'arme combattant à cheval, équipé d'une longue lance et protégé par une armure de plates. La place accordée à l'escrime dite «civile» - c'est-à-dire en vêtements de ville -, et au duel judiciaire semble prépondérante dans un grand nombre de ces textes. Nous devons donc reconnaître que les techniques de combat décrites dans les manuels ne sont pas représentatives de l'escrime médiévale occidentale dans son ensemble, mais 
seulement de quelques traditions ${ }^{19}$. L'objet de la recherche est par conséquent bien plus restreint qu'il n'y paraît. Et cela d'autant plus qu'à l'instar d'autres domaines du savoir médiéval, nous pouvons affirmer que les traités qui nous sont parvenus ne représentent qu'une fraction du savoir accumulé au Moyen Âge.

\section{Les manuels de combat ne se complètent pas systématiquement les uns, les autres}

Nous possédons rarement plusieurs œuvres d'un même maître d'armes ${ }^{20}$. En fait, nombreux sont les manuels difficilement datables, dont l'auteur est resté anonyme et/ou connu simplement grâce à une copie unique. Replacer ces ouvrages dans l'ensemble du corpus et découvrir leur influence réciproque sont souvent des opérations périlleuses. Un lien évident existe cependant entre plusieurs textes allemands (Wierschin 1965; Hils 1985). Néanmoins, les relations qui pouvaient exister entre leurs auteurs sont méconnues. D'autant plus que certains d'entre eux interprètent parfois de manières très différentes un même enseignement ${ }^{21}$. C'est le cas, par exemple, des textes attribués à Peter von Danzig, à Sigmund Ringeck et à Peter Falkner (Tobler 2010: 83-91, 2012: 6-9; Mitchell 2014: 5-18). Il est très difficile, voire dangereux, d'effectuer une recherche simultanée sur plusieurs sources afin d'essayer de combler les vides, les silences ou les interrogations apparues lors de la lecture d'un manuel bien précis.

\section{Les mots sont insuffisants pour décrire un mouvement ou une technique}

La technique de combat est une action d'une extrême complexité puisqu'elle se compose généralement d'un enchaînement de poses et de mouvements mais aussi parce que son déroulement est influencé par des facteurs psychologiques ${ }^{22}$. Pour la décrire de manière relativement complète, il est nécessaire de fournir un très (trop) grand nombre de détails. Quelles sont les positions de départ? Quelle distance sépare les combattants? Quelles sont leurs intentions? Quels sont leurs mouvements et quels déplacements de pieds ces mouvements impliquent? Quelle est la trajectoire empruntée par les lames? Lorsqu'elles s'entrechoquent, à quel endroit a lieu l'impact? Sur le plat ou le tranchant, sur le faible ou le fort de la lame? Quelle est la pression exercée lors d'un liage? Nous pourrions naturellement continuer cette liste, mais ces quelques exemples permettent d'affirmer que le mot ne suffit pas pour décrire dans le détail une technique de combat. Cette situation ne posait évidemment aucun problème aux maîtres d'armes de l'époque puisque leurs textes n'étaient pas destinés à être interprétés sans avoir suivi l'enseignement oral desdits maîtres ${ }^{23}$. De nos jours, comme il n'est plus possible de bénéficier de leurs leçons, l'interprétation de leurs écrits devient un véritable défi.

\section{Le vocabulaire technique utilisé est souvent difficile à interpréter}

La grande majorité des manuels de combat est écrite en langues vernaculaires, le latin n'apparaissant que dans de rares textes ${ }^{24}$. Il semblerait que cette dernière langue ne possédait pas un vocabulaire suffisamment clair et précis pour satisfaire les auteurs. C'est du moins ce que suggère la lecture du Leeds, Royal Armouries, MS. I.33, par l'enchevêtrement 
de termes techniques allemands au texte latin (Cinato \& Surprenant 2009), ou les commentaires de Pietro del Monte (1457-1509) ${ }^{25}$. Ainsi, les auteurs ont besoin d'utiliser un vocabulaire aussi riche que précis. Pierre-Alexandre Chaize a signalé qu'il se divise en trois catégories distinctes: le vocabulaire commun compris de tous, le vocabulaire technique adapté au sujet traité et généralement assimilé par les habitués de la matière et un vocabulaire exclusif réemployant l'ensemble des mots spécifiques à l'auteur (Chaize 2013: 103-118). C'est bien entendu ce dernier type de vocabulaire qui pose le plus de problème et le sens exact de plusieurs mots nous échappe toujours; d'autant plus qu'un même terme est des fois utilisé de manière différente par quelques auteurs (Boffa 2014: 57-59). Ceci étant dit, nous devons être systématiquement attentifs. C'est pourquoi, dans un passage du Ms 3227a, selon que nous comprenons le mot «rechte» comme «(à la) droite» ou comme «juste, idéal», l'interprétation de l'extrait diffère totalement ${ }^{26}$ (Chaize 2013: 105).

\section{La notion de secret}

Le secret entourait l'enseignement des maîtres d'armes ${ }^{27}$. Pour certains, il était simplement hors de question de permettre au commun des personnes de découvrir les subtilités d'un art réservé à la noblesse (Rubboli \& Cesari 2005: 31) ${ }^{28}$. Pour d'autres, les mobiles étaient bien plus pragmatiques: ils avaient conçu leur système de combat après un long apprentissage et il n'aurait pas été sage de dévoiler rapidement le fruit de plusieurs années de pratique et de réflexion - surtout si ceux-ci espéraient en vivre. Ils distillaient alors leur art de manière parcimonieuse; non seulement pour intéresser le plus longtemps possible leurs élèves, mais aussi pour conserver une certaine supériorité sur des disciples trop doués ou des adversaires potentiels ${ }^{29}$. Lorsque nous abordons un texte, nous devons donc nous interroger sur l'ampleur et la valeur du contenu technique qu' il recèle. Il est d'ordinaire impossible de répondre avec certitude à ces deux questions.

\section{Les illustrations jouent un rôle essentiel, mais leur pouvoir est limité}

Le combat est une activité dynamique qui se déroule dans l'espace et dans le temps; il est illusoire de vouloir le restituer par quelques poses statiques (Anglo 2011). De plus que les artistes du Moyen Âge ne disposaient pas des outils nécessaires pour offrir un système de représentation graphique (images, diagrammes ou schémas) riche en informations. Il faut attendre le Xvi siècle pour que les auteurs innovent dans ce domaine ${ }^{30}$. Le lecteur de manuels récents ne doit donc pas espérer y découvrir l'enchaînement des mouvements, les variations de distance, les déplacements des protagonistes et plus particulièrement leurs mouvements de pieds ou la vitesse d'exécution d'une technique (Fermor 1987: 18-32 ${ }^{31}$ ).

Cela ne signifie nullement que ces illustrations sont sans valeur, elles facilitent grandement la compréhension des descriptions de techniques martiales. Cependant il est nécessaire d'établir clairement le rôle que l'auteur leur attribue dans la conception de son œuvre et, le rapport hiérarchique qui peut exister entre le texte et les illustrations. Ces dernières doivent-elles faciliter la compréhension du texte ou celui-ci doit-il expliciter l'image? Il convient ensuite de déterminer le moment privilégié par l'auteur pour figer le temps. S'agit-il du début de l'action, du moment critique (avant le coup de grâce) ou d'une phase clef de l'action? C'est à ce prix que les illustrations nous livreront une partie de leur secret. 


\section{Les illustrations sont parfois maladroites voire erronées}

Le travail des illustrateurs est parfois ambivalent (Boffa 2014: 60-63) et l'« erreur» facilement décelable: comme lorsque Hans Talhoffer parle du maniement d'un Messer alors que le combattant est représenté avec une épée ${ }^{32}$. Ces «manquements» ne sont pas toujours aisément repérables. Pour s'en convaincre, il suffit de comparer comment la garde «vom Tag» est illustrée dans de nombreux manuels. Les multiples fluctuations que nous relevons sont-elles liées au style de chacun des auteurs ou à la variation des illustrations du geste?

\section{Limpossibilité de valider le travail de reconstitution}

Imaginons qu'un chercheur ait judicieusement choisi une source historique riche en informations, qu'il ait élaboré une méthode de travail consciencieuse et qu'il ait réussi à éviter les écueils énumérés ci-dessus. Un dernier problème subsiste. Comment vérifier la justesse de son travail de reconstitution?

\section{Le critère d'efficacité}

Lorsqu'il s'agit de valider le résultat d'une recherche, il est souvent question d'efficacité. Voici l'une des procédures suggérées:

«So how do we validate offensive action? There are four tests that we use in the Academy of Historical Arts:

1. does it match the source?

2. does it work in drilling?

3. does it work in cutting?

4. does it work in sparring ${ }^{33}$ ?»

Il n'est pas nécessaire de la détailler. Constatons simplement que seule la première de ces étapes lie la technique reconstituée à la source. C'est étonnant. Personnellement, nous ne comprenons pas comment la manière dont une technique est exécutée ou utilisée par le pratiquant peut exprimer le succès ou l'échec de la reconstitution historique. Et quand nous interrogeons l'auteur sur le procédé utilisé pour vérifier si la technique correspond bien à la source, sa réponse est bien trop naïve:

«So, if the technique looks like illustrations from the sources and if its performance matches the descriptive text in the source, then the technique passes the first test ${ }^{34}$.»

Ce ne sont pas tant les méthodes élaborées sur le principe d'efficacité qui doivent être mises en cause, mais ce principe même. En effet, l'escrime médiévale se pratiquait dans deux environnements distincts, agonistique et antagonistique, traduis généralement dans les textes par une escrime ludique (Schimpf) et une escrime sérieuse (Ernst) (Jaquet 2012 : 77-87). Le lecteur n'est pas toujours averti de l'environnement dans lequel une technique doit être exercée. Entre autres, Johannes 
Lecküchner passe régulièrement d'une escrime à l'autre dans son manuel, et de temps en temps dans une même description ${ }^{35}$. Cette situation particulière rend encore plus ardu le jugement de l'efficacité d'un mouvement. Ainsi, comme le précise Daniel Jaquet, «évaluer un geste nécessite non seulement de comprendre son cadre d'application, mais aussi de reconstruire les critères contemporains de perception d'un geste "réussi" » (Jaquet \& Kiss 2015 : 56-72). Dès lors, une manière d'exécuter un mouvement qui nous semblerait moins efficace ne pourrait pas être écartée au profit d'une autre qui nous paraîtrait imparable. Il s'agirait d'un jugement subjectif qui n'a rien de scientifique.

Enfin, vouloir juger la validité d'une reconstruction sur la base d'un critère d'efficacité implique, du moins implicitement, que l'auteur d'un manuel de combat posséde obligatoirement un talent supérieur à ses congénères. Malheureusement, les biographies des maîtres d'armes sont souvent très mal connues et, à quelques exceptions près, rien ne nous permet d'affirmer que ceux-ci étaient systématiquement de redoutables combattants $^{36}$. Si nous acceptons l'idée que certains d'entre eux pouvaient être d'un niveau relativement commun, le critère d'efficacité n'aura plus de sens.

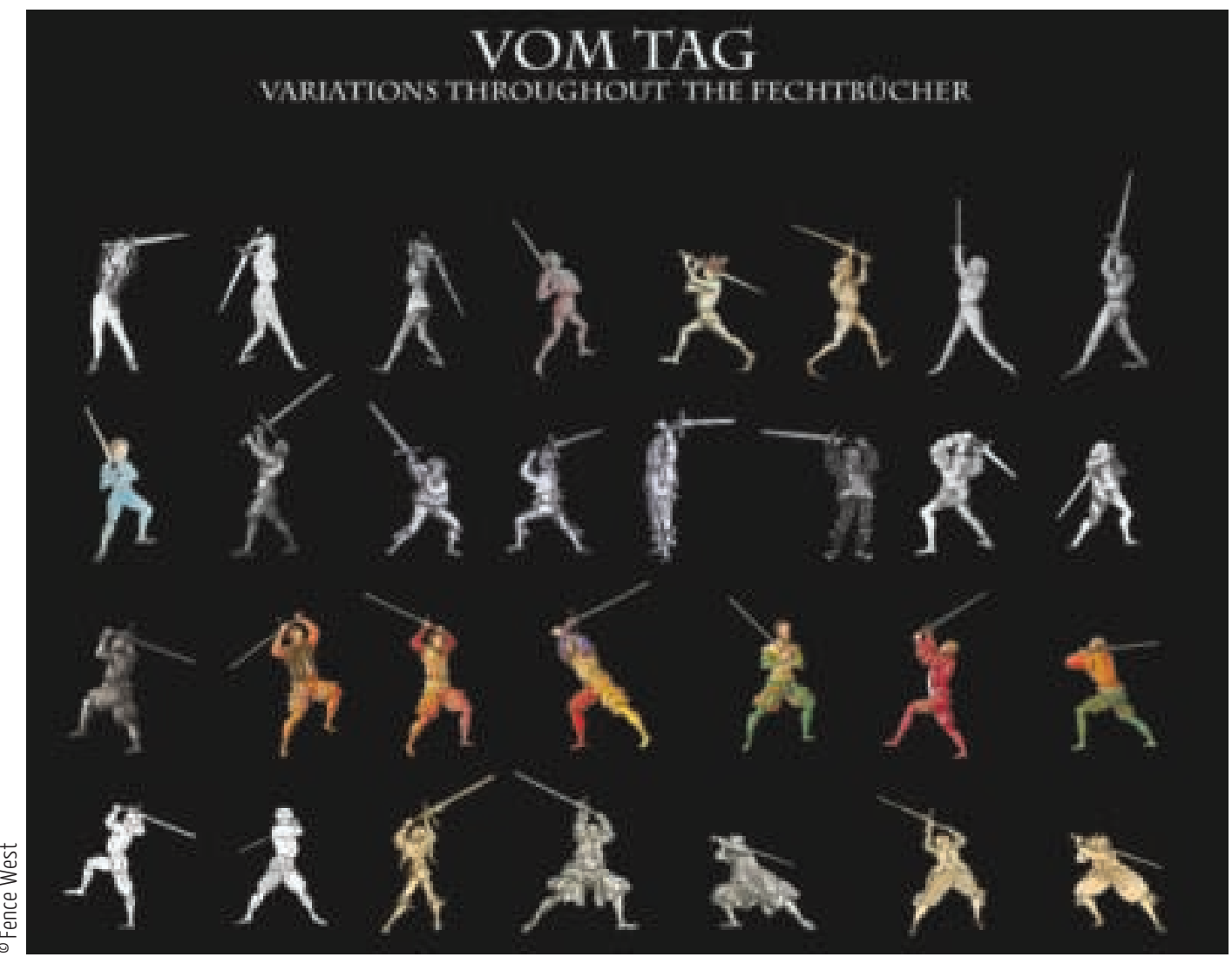

\section{Variations de la garde vom Tag}

Ce tableau, réalisé par un amateur dont nous n'avons pas pu retrouver le nom, montre qưune même garde, dans ce cas le garde vom Tag («Du Jour »), peut être représentée de manières bien distinctes. Ces variations s'expliquent facilement: interprétation personnelle de cette garde par le maître d’arme, moment de l'action différent d'une représentation à l'autre ou travail plus ou moins négligé de la part de l'illustrateur. 


\section{La comparaison avec d'autres arts martiaux}

Comparer les résultats obtenus avec le curriculum d'autres arts martiaux, généralement originaires d'Asie, serait un autre moyen de valider la recherche ${ }^{37}$. C'est une démarche douteuse. Les systèmes de combat, qu'ils soient orientaux ou occidentaux, se concentrent sur les mêmes points faibles (articulations, nerfs, parties génitales, yeux, etc.) ou létaux (cour, artères, ventre, etc.). Il est donc logique de trouver des ressemblances entre des systèmes fondés sur des principes identiques. Matthew Galas (1997: 20-47) a explicité les nombreuses similitudes qui existent entre l'escrime médiévale occidentale et l'art du sabre japonais. Cependant, le boxeur et le karatéka donnent leurs coups de poing de manière bien différente. De même, les coupes au sabre du kendo ka, de l'escrimeur moderne et du pratiquant de HEMA ne se ressemblent guère. En fin de compte, que nous pointions les similitudes ou les différences, nous avons du mal à concevoir une procédure permettant de valider le travail de nos chercheurs. Cela ne signifie évidemment pas que la connaissance d'un autre art martial soit totalement inutile, elle peut être une source d'inspiration et un stimulant pour la recherche. Néanmoins, il faut cependant prendre soin de ne pas oublier que chaque système de combat possède des spécificités qui lui sont propres. Dans le cadre d'une recherche historique, il n'est pas autorisé d'importer telle quelle une technique d'un art à un autre.

\section{Pouvons-nous parler d'archéologie expérimentale?}

Certaines personnes associent ces tentatives de reconstitution à de l'archéologie expérimentale (Coles1979; Ferguson 2010 ; Millson 2011). Pour qu'il en soit ainsi, nous devrions disposer de données permettant de proposer une hypothèse qui pourrait être vérifiée ou non par l'expérimentation (Kelterborn 2005 : 120-124; Lammers-Keijsers 2005 : 18-24). Dans le cas de notre étude, les données seraient les informations présentes dans les manuels et l'hypothèse serait un mouvement reconstitué, dont l'authenticité ou l'historicité devrait être vérifiée par l'expérimentation. C'est bien ici que se pose le problème. Comment vérifier l'authenticité d'un geste? Comment savoir si la technique qui vient d'être élaborée est précisément celle qui était exécutée par l'auteur? Nous ne disposons d'aucun élément de référence proposant d'effectuer cette vérification puisque nous savons que des critères comme l'efficacité ou la similitude avec une technique présente dans un autre art martial ne sont pas suffisants. 


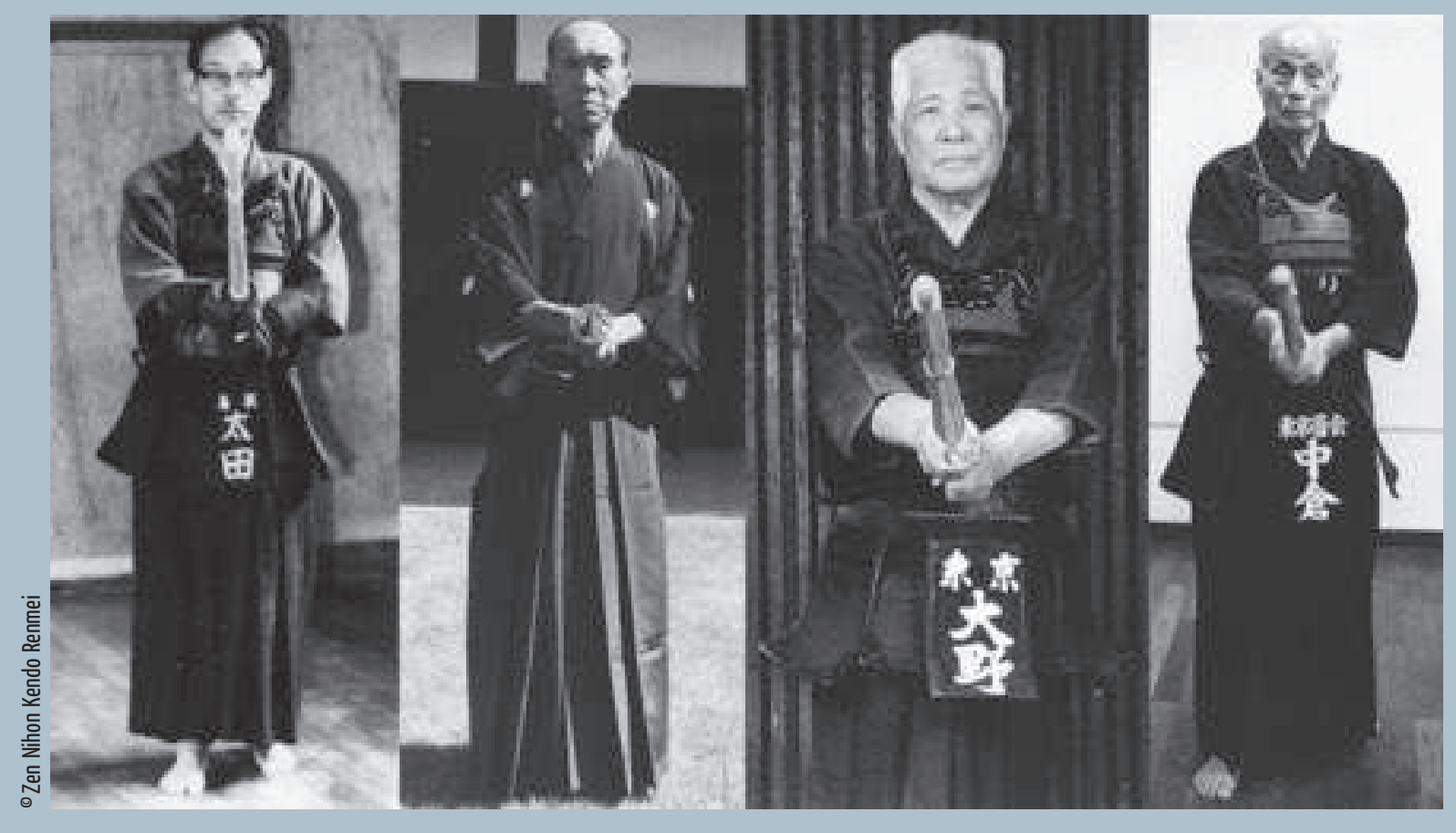

\section{Variations de la garde moyenne (chîdan no kamae)}

L'enseignement officiel décrit la garde chûdan no kamae ainsi: « Standing upright, the right foot is forward slightly, with the front of the left foot placed in line with the right heel. The shinai (bamboo sword) is held with the right hand just below the tsuba (sword guard), and with the left hand at the tsuka-gashira (end of the shinai hilt), while the extension of the kensen (tip of the shinai) points between the opponent's eyes.» (All Japan Kendo Federation 2011 Japanese-English Dictionary of Kendo. Tokyo, $2^{e}$ éd.: 22).

Bien que cette défnition soit très précise, chaque maître prend cette position d'une manière qui lui est propre. Le premier (Ôta sensei) utilise une variante ancienne de cette garde connue sous le nom de seigan no kamae. La pointe du sabre n'est pas dirigée vers la gorge ou les yeux de l'adversaire, mais est levée bien plus haut. Le second (non identifié) utilise cette même garde avec un vrai sabre. Le troisième (Ôno sensei) utilise la version orthodoxe. Le dernier (Nakakura sensei) ne se tient pas face à l'adversaire, mais tourne légèrement son corps vers la gauche (hanmi). La pointe de son sabre est aussi plus basse. Ces variations, qui défnissent le style et la compréhension de cette garde par chacun de ces maîtres, ne sont pas explicitées dans les textes et sont transmises uniquement oralement. Nous laissons au lecteur la tâche d'imaginer comment les illustrateurs médiévaux, avec leur maîtrise imparfaite de la perspective, auraient pu rendre graphiquement visible et intelligible de telles subtilités. 
L'escrime médiévale européenne était aussi développée que raffinée mais, malheureusement, l'interruption séculaire de son enseignement a causé des dommages irréparables dans la transmission de cet art. L'historien qui s'attaque à sa redécouverte dispose des manuels de combat, une source historique du plus grand intérêt. Cependant, il est important de souligner que le savoir conservé n'est pas représentatif de l'ensemble de l'escrime médiévale. Il est limité tant dans le temps - XIv et $\mathrm{Xv}^{\mathrm{e}}$ siècles - que dans l'espace - principalement les terres d'Empire.

Le procédé d'enregistrement de ces informations dans ces manuels est bien plus problématique. Les auteurs ne nous ont transmis qu'une partie de leur enseignement. Volontairement, car le secret l'entourait. Involontairement, parce que le mot et l'image ne peuvent convoyer l'idée de mouvement ni décrire avec précision une action complexe comme la technique de combat avec tous ses éléments biomécaniques, techniques ou psychologiques - un problème auquel nous ne sommes pas moins confrontés avec les moyens filmiques (De France 2010 : 220-222).

Tout cela signifie-t-il qu'entreprendre des recherches dans ce domaine soit impossible? Bien sûr que non. Un grand nombre de passionnés - escrimeurs désireux d'explorer les racines de leur art, historiens amateurs et professionnels ou simples amoureux du Moyen Âge -ont prouvé le contraire. Grâce à leurs efforts, il est actuellement possible de se faire une idée relativement précise du maniement de l'épée aux xIV et Xve siècles. Il est toutefois nécessaire de réaliser quelles sont les limites de ces découvertes.

Lorsque le maître d'armes a choisi de s'étendre sur les principes théoriques qui structurent son savoir, les principes généraux se laissent entrevoir à la lecture des manuels, même si toutes leurs subtilités peuvent encore nous échapper. Le texte de Johannes Lecküchner est suffisamment important et détaillé pour que nous puissions les y reconnaître par exemple (Forgeng 2015).

Redécouvrir une technique de combat est bien plus problématique. Mais si nous disposons d'une ou de plusieurs sources suffisamment détaillées et d'une méthode d'investigation sérieuse c'est évidemment possible. Nous pensons plus particulièrement à la tradition allemande et notamment à Johannes Liechtenauer que nous pouvons interpréter grâce à de nombreux glossateurs (Tobler 2004, 2007, 2010, 2012; Zabinski 2010) ou à l'enseignement de Fiore dei Liberi qui nous est parvenu au travers de multiples manuscrits (Malipiero 2006).

À notre avis, il n'est pas possible d'aller au-delà de la résurrection d'une technique. Les manuels de combat ne sont pas assez explicites pour que nous puissions espérer y découvrir le style propre à un maître du passé; non pas de comprendre le déroulement ou la mécanique d'une technique, mais de saisir comment cette technique a été interprétée et exécutée par un individu particulier. À ce stade, il ne s'agit plus d'interpréter un texte, mais d'imiter à l'identique un modèle, un modèle qui n'est plus.

Bien que limités dans leurs perspectives, ces travaux peuvent-ils être qualifiés de scientifiques? Il est difficile de répondre à cette question, d'autant plus que ce domaine de recherche est particulièrement actif et que de nouvelles approches méthodologiques sont constamment proposées. Quoi qu'il en soit, le sérieux de certains de ces chercheurs ne peut être mis en doute. Pourtant, quelle que soit la qualité de leur travail, il est actuellement impossible d'en vérifier l'authenticité ou l'historicité. 


\section{NOTES}

Photo d'ouverture: Combattants équipés de copies d'armes anciennes. Certains pratiquants préfèrent utiliser de fidèles imitations des anciennes armes offensives et défensives. Cela leur permet de prendre réellement conscience du poids et de la maniabilité des épées de l'époque ainsi que des contraintes imposées par le port de l'armure. Il est important de ne pas confondre cette démarche avec celle qui consiste simplement à revivre un Moyen Âge que les anglophones appellent le reenactment.

1. Dans le monde anglo-saxon, on utilise les expressions Historical European Martial Arts (HEMA), European Medieval Martial Arts (EMMA) ou Western Martial Arts (WMA).

2. Avant d'entrer dans le vif du sujet, précisons que dans cet article nous nous intéresserons principalement au combat à l'épée.

3. Voir le site de l'Association for Renaissance Martial Arts "About the ARMA», www.thearma.org/about.htm\#.

4. Il n'en existe toujours pas d'inventaire complet. À défaut, on consultera Rainer Leng (2008) et Daniel Jaquet (2012: 209-212).

5. Voir Franck Cinato et André Surprenant (2009).

6. Nous pensons aux travaux de Paulus Hector Mair (1517-1579) et de Joachim Meyer (1537-1571).

7. Le De Dignoscendis Hominibus de Pietro del Monte (1492) ou le Das Landshuter Ringerbuch imprimé par Hans Wurm dans les années 1490.

8. Ce qui s'apparente aux kata et aux suburi dans les arts martiaux japonais.

9. Curieusement, les manuels sont extrêmement discrets sur les aspects psychologiques du combat.

10. Pour information, voici les différentes phases qui composent une attaque en escrime japonaise moderne (kendô) : 1. Prendre une garde (kamae); 2 . Tester la garde de l'adversaire et créer une faiblesse dans celleci (seme-ai); 3. Reconnaître cette faiblesse et choisir la technique qui nous permettra d'en tirer profit; 4 . Lancer une attaque techniquement correcte (yukô datotsu); 5. Maintenir un état de vigilance afin de pouvoir répéter ce processus si l'attaque n’a pas été décisive (zanshin).

11. Nous le remarquons facilement tant dans de nombreux sports que dans les arts martiaux actuels.

12. Et, dans certains cas, par la manière dont ces techniques sont exécutées (le style du maître).

13. Doit-on préciser que plus le curriculum est large, plus les chances d'en comprendre la logique et d'en découvrir les finesses seront élevées?
14. Soulignons la subtilité qui différencie experimenting d'experiencing.

15. Voir le site de Jamie Acutt «Paleoeskirmology Pré-scientific Martial Arts», eskirmology.co.uk/ paleoeskirmology/index.

16. Il n'existe toujours pas de synthèse technique sur l'escrime médiévale européenne. L'ouvrage de John Clements est totalement dépassé (1998).

17. Les ouvrages de Christian Henry Tobler représentent une bonne introduction à la tradition allemande (2004, 2007).

18. C'est pourquoi nous restons dubitatifs sur la qualité du travail des « chercheurs » qui « redécouvrent » l'escrime Viking (VIII ${ }^{\mathrm{e}}-\mathrm{XI}^{\mathrm{e}}$ siècles) à l'aide de sources des XIV et XV siècles.

19. Pour caricaturer à l'extrême, nous pourrions affirmer que les manuels offrent un aperçu de l'escrime pratiquée au Bas Moyen Âge dans les terres centrales de l'Empire par une partie de la société de l'époque difficilement identifiable et à utiliser dans des circonstances bien précises...

20. Fiore dei Liberi (fin $\mathrm{XIV}^{\mathrm{e}}$-début $\mathrm{Xv}^{\mathrm{e}}$ siècle) et Hans Talhoffer ( $x v^{e}$ siècle) étant deux exceptions notables.

21. Celui de Johannes Liechtenauer.

22. Voir ci-dessus

23. Ils étaient d'ailleurs conscients de cet état des choses : «Auch merke das und wisse das man nicht gar eygentlich und bedewtlich von dem fechten mag sagen und schreiben ader aus legen als man is wol mag czeigen und weisen mit der hant », c'est-à-dire "Sache et note aussi que l'on ne peut pas vraiment parler de l'escrime de manière significative ou l'expliquer avec l'écrit comme certain l'aimerait. Tu peux seulement la montrer et l'enseigner par le geste. » (Nuremberg, Germanisches Nationalmuseum, Ms 3227a, f. 15 r.).

24. Le latin apparaît dans le Leeds, Royal Armouries, MS. I.33 ; le Florius, De Arte Luctandi de Fiore dei Liberi et dans certains textes de Pietro del Monte. 
25. Pietro del Monte, De singulari certamine sive dissensione deque veterum recentiorumque ritu ad Carolum Hyspaniarum principem et Burgundie archiducem libri tres, Milan, 1509, liv. I, Prologus, Sig. AA iv vº Pietro del Monte, Petri Montii exercitiorum, atque artis militaris collectanea, Milan, 1509, Liv. I, Chap. I.

26. Nuremberg, Germanisches Nationalmuseum, Ms 3227 , f. $78 \mathrm{r}$.

27. Ainsi Peter von Danzig ( $\mathrm{xv}^{\mathrm{e}}$ siècle) nous apprend que Johannes Liechtenauer a transmis son art à l'aide de « mots secrets »: «So hat er die selbig kunst igleich besunder lassen schreiben mit verporgen vnd verdackten worten, Dar umb das sÿ nit yderman vernemen noch versteen sol, als du sÿ her nach geschriben wirst vinden » soit «il [Liechtenauer] a écrit sur cet art avec des mots cachés et secrets, ainsi tout le monde ne pourra pas le saisir et le comprendre. » (Rome, Biblioteca dell'Accademia Nazionale dei Lincei e Corsiniana, Cod. 44 A 8, f. 3 r.).

28. Filippo Vadi, qui s'est inspiré de Fiore dei Liberi, partage cette vision élitiste (Porzio \& Mele 2002: 32-35).

29. «Das ein meister im alwegen sol etwas behalten das er seine schüler nit leren sol. » Soit: « qu'un maître, en toute matière doit garder quelque chose qu'il ne doit pas enseigner à son élève.» (Pauli 1866: 199).

30. Les traités de Camillo Agrippa et d'Henry de SainctDidier marquent un tournant dans la représentation des mouvements de l'escrimeur (Agrippa 1553; De Sainct-Didier 1573; Anglo 2011 : 25, 31-35).

31. Sur les limites du pouvoir informatif de l'image isolée dans le cas de la danse.

32. Copenhague, Det Kongelige Bibliotek, MS Thott. $290.2^{\circ}$, f.79 r.

33. Voir le site Encased in Steel, «Validating what we do in Martial Arts by Keith Farell », www.encasedinsteel. co.uk/2013/05/03/validating-what-we-do-in-martial-arts/.

34. Ibid.

35. Par exemple Munich, Bayerische Staatsbibliothek, Cgm 582, f. 22v.

36. Être l'auteur d'un manuel de combat n'est évidemment pas un argument suffisant. Sinon, en toute logique, tous les auteurs contemporains qui partagent leurs réflexions et leurs travaux sur les HEMA devraient aussi être considérés comme des escrimeurs de haut niveau.

37. Voir le site de HEMA Alliance, Jason Vail, « The Role of other Martial Arts in Validating HEMA Interpretations», hemaalliance.com/learning/articles/the-role-of-othermartial-arts-in-validating-hema-interpretations/. ne peut pas vraiment parler de l'escrime de manière significative ou l'expliquer avec l'écrit comme certain l'aimerait. Tu peux seulement la montrer et l'enseigner par le geste. » (Nuremberg, Germanisches Nationalmuseum, Ms 3227a, f. 15 r.). 


\section{RÉEÉRENCES}

Agrippa, C. 1553 Trattato di scientia d'arme con un dialogo di filosofia. Rome: A. Blado.

Alderson, K. 2010 "On the Art of Reading. An Introduction to Using the Medieval German "Fightbooks" » in G. D. Mele dir. In the Service of Mars. Proceedings from the Western Martial Arts Workshop, 1999-2009. Wheaton: Freelance Academy press: vol. I.

Anglo, S. 2000 The Martial Arts of Renaissance Europe. Londres et New Haven: Yale University press.

Anglo, S. 2011 L'escrime, la danse et l'art de la guerre. Le livre et la représentation du mouvement. Paris: Bibliothèque nationale de France.

Boffa, S. 2014 Les manuels de combat. Fechtbücher et Ringbücher. Turnhout: Brepols.

Capwell, T. 2012 The Noble Art of the Sword. Fashion and Fencing in Renaissance Europe, 1520-1630. Londres: Wallace Collection.

Castle, E. 2003 Schools and Masters of Fencing. From the Middle Ages to the Eighteenth Century. New York: Dover Publications.

Chaize, P.-A. 2013 «Des mots aux gestes. Le rôle du texte et du vocabulaire dans l'expérimentation historique », Staps 101: 103-118.

Cinato, F. \& A. Surprenant 2009 Le livre de l'art du combat (Liber de arte dimicatoria). Commentaires et exemples. Édition critique du Royal Armouries MS. I.33. Paris: CNRS Éditions.

Clements, J. 1998 Medieval Swordsmanship. Illustrated Methods and Techniques. Boulder: Paladin press.

Cohen, R. 2002 By the Sword. Gladiators, Musketeers, Samurai Warriors, Swashbucklers and Olympians. Londres: Random House.

Colciago, S. 2014 «A Glimpse of these Forgotten Arts. Metodologie di recupero del sapere marziale», Antropologia e Teatro 5: 119-161.

Coles, J. 1979 Experimental Archaeology. Londres, New York: Academic press.

DeFrance, C. 2010 «Lanalyse praxéologique. Composition, ordre et articulation d'un procès», TechniquesECulture 54-55 (1): 220-222.

De Sainct-Didier, H. 1573 Traicté contenant les secrets du premier livre sur l'espée seule, mère de toutes armes, qui sont espée, dague, cappe, targue, bouvlier, rondelle, l'espée deux mains... Paris: J. Dalier.

Ferguson, J. R. dir. 2010 Designing Experimental Research in Archaeology. Examining Technology Through Production and Use. Boulder: University Press of Colorado.

Fermor, S. 1987 «On the Question of Pictorial "Evidence" for Fifteenth-Century Dance Technique », Dance Research. The journal of the Society for Dance Research 5 (2): 18-32.

Forgeng, J. L. 2015 The Art of Swordsmanship by Hans Lecküchner. Suffolk, Rochester: Boydell \& Brewer.

Galas, M. 1997 « Kindred Spirits. The Art of the Sword in Germany and Japan », Journal of Asian Martial Arts $6(3): 20-47$.

Hand, S. 2005 «The Importance of Principles over Techniques» in B. R. Price dir., Teaching \& Interpreting Historical Swordsmanship. Union City: The Chivalry Bookshelf.

Hils, H.-P. 1985 Meister Johann Liechtenauers Kunst des langen Schwertes. Francfort-sur-le-Main, New York: P. Lang.

Jaquet, D. 2012 «Entre éducation et festivité. Schirm-und Fechtschulen dans l'ancienne confédération suisse, $\mathrm{XV}^{\mathrm{e}}-\mathrm{XVI}^{\mathrm{e}}$ siècle» in O. Meuwly \& N. Gex dir. Duel et combat singulier en Suisse romande. De l'antiquité au $x x^{e}$ siècle. Actes du colloque des 7 et 8 mai 2010. Bière: Cabedita.

Jaquet, D. dir. 2012 Lart chevaleresque du combat. Le maniement des armes à travers les livres de combat (XIve$X I^{e}$ siècles). Neuchâtel: Éditions Alphil: Presses universitaires suisses.

Jaquet, D. et al. 2015 «Historical European Martial Art. A Crossroad between Academic Research, Martial Heritage Re-Creation and Martial Sport Practices », Acta Periodica Duellatorum 3 : 5-35.

Jaquet, D. \& D. Kiss 2015 «L'expérimentation du geste martial et du geste artistique: regards croisés», Ephaïstos 4 (1): 56-72.

Kelterborn, P. 2005 «Principles of Experimental Research in Archaeology », EuroREA 2: 120-124. 
Lacaze, P. 1991 En garde. Du duel à l'escrime. Paris: Gallimard.

Lammers-Keijsers, Y. M. J. 2005 «Scientific Experiments: A Possibility? Presenting a General Cyclical Script for Experiments in Archaeology », EuroREA 2: 18-24.

Leng, R. 2008 «Fecht-und Ringbücher» in Katalog der deutschsprachigen illustrierten Handschriften des Mittelalters, Band 4/2, Lieferung 1/2, 38. Munich: C. H. Beck.

Lindholm, D. \& P. Svard 2003 Sigmund Ringeck's Knightly Art of the Longsword. Boulder, Paladin press.

Malipiero, M. 2006. Il Fior di battaglia di Fiore dei Liberi da Cividale, Il Codice Ludwig XV 13 del J. Paul Getty Museum. Éditions Ribis.

Malipiero, M. 2011 «La scherma storica» in F. Lodà dir., La bottega dello storico. Le metodologie della ricerca nella scherma storica. San Marino Città: Il Cerchio.

Millson, D. C. E. 2011 Experimentation and Interpretation. The Use of Experimental Archaeology in the Study of the Past. Oxford, Oakville: Oxbow Books.

Mitchell, R. 2014 « There is no One True Art of the Sword» in M. A. Cramer dir., «Can These Bones Come to Life?» Insights from Reconstruction, Reenactment, and Re-creation, Volume II : High in Protean Content. Wheaton: Freelance Academy press.

Oakeshott, E. 2000 European Weapons and Armour. From the Renaissance to the Industrial Revolution Woodbridge. Suffolk, Rochester: Boydell \& Brewer.

Ohya, M. 2000 «The Technical and Psychological Methodology of Kendô» in Budo Studies. An Anthology of Research into Budo in the $21^{\text {st }}$ Century. Katsuura: Institute of Budo/Sports Science Research, International Budo University.

Pauli, J. 1866 Schimpf und Ernst. Stuttgart Litterarischen Vereins.

Porzio, L. \& G. Mele, 2002 Arte gladiatoria dimicandi, 15th Century Swordsmanship of Master Filippo Vadi. Union City: The Chivalry Bookshelf.

Price, B. R. 2008 The Sword in Two Hands. A Full-Color Training Guide for the Medieval Longsword based on Fiore dei Liberi's Fior di Battaglia. Union City: The Chivalry Bookshelf.

Rubboli, M. \& L. Cesari 2005 Flos Duellatorum, Manuale di arte del combattimento del xv secolo. San Marino Città : Il Cerchio.

Rubboli, M. 2011 « Metodologie di ricerca nell'ambito della scherma storica con riferimento ad ambiti temporali diversi. Il caso delle scuole italiane alla fine del Medioevo e nel Rinascimento e il caso della scherma nell'Antichita classica a confronto » in F. Lodà dir., La bottega dello storico. Le metodologie della ricerca nella scherma storica. San Marino Città: Il Cerchio.

Tobler, C. H. 2004 Fighting with the German Longsword. Union City: The Chivalry Bookshelf.

Tobler, C.H. 2007 Secrets of German Medieval Swordsmanship. Sigmund Ringeck's Commentaries on Master Liechtenauer's Verse, $2^{e} e ́ d$. . Union City: The Chivalry Bookshelf.

Tobler, C. H. 2010 «Introduction to the Von Danzig Fechtbuch» in C. H. Tobler, In Saint George's Name. Wheaton, Freelance Academy press.

Tobler, C. H. 2012 Captain of the Guild. Master Peter Falkner's Art of Knightly Defense. Wheaton, Freelance Academy press.

Walczak, B. 2006 «The AGISE Research Method» in F. Cognot dir., Maitres $\&$ techniques de combat à la fin du Moyen Âge et au début de la Renaissance. Paris: AEDEH.

Wierschin, M. 1965 Meister Johann Liechtenauers Kunst des Fechtens. Munich: C. H. Beck.

Windsor, G. 2005 « Getting Started in Historical Swordsmanship» in B. R. Price dir., Teaching \& Interpreting Historical Swordsmanship. Union City: The Chivalry Bookshelf.

Windsor, G. 2012 Veni Vadi Vici. A transcription, translation and commentary of Philippo Vadi's De Arte Gladiatoria Dimicandi. Union City: The Chivalry Bookshelf.

Żabiński, G. 2010 The Longsword Teachings of Master Liechtenauer. The Early Sixteenth Century Swordsmanship comments in the "Goliath» Manuscript. Torun: Wydawnictwo Adam Marszarek.

Żabiński, G. et al. 2012 A Falchion: Langes Messer Fencing Treatise by Johannes Lecküchner (1482). Hambourg. 


\section{POUR CITER CET ARTICLE}

Boffa, S. 2017 «Réflexions sur la résurrection de l'escrime médiévale occidentale. Méthode, objectifs et limites», in G. Bartholeyns \& F. Joulian, Le corps instrument, Techniques\&Culture 62: 166-187.

\section{RÉSUMÉ}

\section{Réflexions sur la résurrection de l'escrime médiévale. Méthode, objectifs et limites}

Depuis quelques années, des associations se constituent afin de ressusciter les anciennes méthodes occidentales de combat. Cette entreprise est particulièrement difficile à mener car la majorité de ces traditions martiales a disparu au Xvi siècle. Ces chercheurs ont néanmoins développé différentes méthodes expérimentales comprenant tant l'étude approfondie des sources historique que le maniement d'un équipement semblable à celui utilisé par les combattants de jadis. Le sérieux de ces travaux permet de penser qu'il est effectivement possible de faire revivre un art martial dont la pratique a cessé depuis fort longtemps. Nous ne partageons pas cet optimisme. Dans cet article nous essayerons de montrer que, s'il est possible de reproduire un mouvement proche de celui qui était exécuté il y a plusieurs siècles, les manuels de combat ne sont pas assez explicites pour que nous puissions espérer y découvrir le véritable style des maîtres du passé.

\section{ABSTRACT}

\section{Thoughts on the Revival of the European Medieval Swordsmanship. Method, Objectives and Limitations}

Recently, associations have been formed with the ambition to revive the old Western methods of combat. This work has been particularly difficult to carry out because most of these martial traditions have disappeared in the sixteenth century. However, the researchers have developed various experimental methods including both an in-depth study of historical sources and the handling of equipment similar to the one used by warriors of the past. The depth of these investigations could lead us to believe that it is indeed possible to revive a martial art whose practices have ceased long ago. We do not share this optimism and in this paper we will try to show that it is possible to reproduce a movement similar to, but not exactly that which was executed several centuries ago. We must keep in mind, that the old manuals are not explicit enough for us to hope to discover the true style of the Masters of the past.

\section{MOTS CLÉS}

Art martial, escrime, kendo, archéologie expérimentale, reconstitution historique, manuel de combat, Fechtbuch

\section{KEYWORDS}

Martial art, swordsmanship, kendo, experimental archaeology, re-enactment, fighting manual, Fechtbuch 